\title{
The WASP-South search for transiting exoplanets
}

C. Hellier ${ }^{1}$, D. R. Anderson ${ }^{1}$, A. Collier Cameron ${ }^{2}$, M. Gillon ${ }^{3}$, M. Lendl ${ }^{4}$, T. A. Lister ${ }^{5}$, P. F. L. Maxted ${ }^{1}$, D. Pollacco ${ }^{6}$, D. Queloz ${ }^{4}$, B. Smalley ${ }^{1}$, A. H. M. J. Triaud ${ }^{4}$ and R. G. West ${ }^{7}$

${ }^{1}$ Astrophysics Group, Keele University, UK

${ }^{2}$ SUPA, Physics and Astronomy, University of St. Andrews, UK

${ }^{3}$ Institut d'Astrophysique, Université de Liège, Belgium

${ }^{4}$ Observatoire astronomique de l'Université de Genève, $\mathrm{CH}$

${ }^{5}$ Las Cumbres Observatory, Santa Barbara, USA

${ }^{6}$ Astrophysics Research Centre, Queen's University, Belfast, UK

${ }^{7}$ Dept of Physics and Astronomy, University of Leicester, UK

Abstract. Since 2006 WASP-South has been scanning the Southern sky for transiting exoplanets. Combined with Geneva Observatory radial velocities we have so far found over 30 transiting exoplanets around relatively bright stars of magnitude 9-13. We present a status report for this ongoing survey.

\section{WASP-South}

By 2004 several groups were pursuing systematic surveys for transiting exoplanets, including HAT (Bakos et al. 2004), OGLE (Udalski et al. 2002), TrES (O'Donovan et al. 2006) and XO (McCullough et al. 2006), and the WASP consortium had begun operating a "SuperWASP" camera array on La Palma in the Northern hemisphere (Pollacco et al. 2006). In the South the OGLE project were finding planets around stars of magnitude 15-16, but there was a clear opportunity for WASP's wide-field, bright-sky strategy, aimed at stars of magnitude 9-13.

Funding from consortium universities was secured in 2004, leading to the construction of WASP-South at the South African Astronomical Observatory, closely copying Pollacco's design for the La Palma SuperWASP. Routine robotic operations commenced in March 2006. WASP-South is based around an array of 8 cameras, each with a 200$\mathrm{mm} \mathrm{f} / 1.8$ Canon lens backed by an $e 2 v 2048 \times 2048$ Peltier-cooled chip. Each camera covers $7.8^{\circ} \times 7.8^{\circ}$, with $14^{\prime \prime}$ pixels, so that the array covers $15^{\circ} \times 30^{\circ}$. A broad-band filter gives a $400-700 \mathrm{~nm}$ bandpass.

For the first two years WASP-South covered a "zenith strip" centred at $-32^{\circ}$, but skipping fields in the galactic plane that are too crowded. We typically raster 8-10 fields at a time with a cadence of $\sim 10$ mins, taking two 30 -sec exposures at each pointing. For the next two years WASP-South covered an equatorial strip centred at $-8^{\circ}$, partially overlapping with the Northern SuperWASP, while we are currently covering the far South with fields centred at $-56^{\circ}$.

The data (approx 40 GB per night) are transferred on magnetic tape to Keele to be processed with the WASP pipeline. The data are then accumulated in an archive at Leicester where they are searched for candidate transits (see Collier-Cameron et al. 
EPJ Web of Conferences

Table 1: The WASP-South planets

\begin{tabular}{lllllll}
\hline Name & Position & Star & $V$ mag & $\begin{array}{l}\text { Period } \\
\text { (days) }\end{array}$ & $\begin{array}{l}\text { Mass } \\
\text { (Jup) }\end{array}$ & $\begin{array}{l}\text { Radius } \\
\text { (Jup) }\end{array}$ \\
\hline WASP-4 & $2334-42$ & G7V & 12.6 & 1.34 & 1.12 & 1.42 \\
WASP-5 & $2357-41$ & G4V & 12.3 & 1.63 & 1.64 & 1.17 \\
WASP-6 & $2312-22$ & G8 & 12.4 & 3.36 & 0.50 & 1.22 \\
WASP-7 & $2044-39$ & F5V & 9.5 & 4.95 & 0.96 & 0.92 \\
WASP-8 & $2359-35$ & G6 & 9.9 & 8.16 & 2.24 & 1.04 \\
WASP-15 & $1355-32$ & F5 & 10.9 & 3.75 & 0.54 & 1.43 \\
WASP-16 & $1418-20$ & G3V & 11.3 & 3.12 & 0.86 & 1.01 \\
WASP-17 & $1559-28$ & F6 & 11.6 & 3.74 & 0.49 & 1.74 \\
WASP-18 & $0137-45$ & F9 & 9.3 & 0.94 & 10.4 & 1.17 \\
WASP-19 & $0953-45$ & G8V & 12.3 & 0.79 & 1.15 & 1.31 \\
WASP-20 & & & 10.7 & 2.4 & & \\
WASP-22 & $0331-23$ & & 12.0 & 3.53 & 0.56 & 1.12 \\
WASP-23 & $0644-42$ & K1V & 12.7 & 2.94 & 0.87 & 0.96 \\
WASP-25 & $1301-27$ & G4 & 11.9 & 3.76 & 0.58 & 1.22 \\
WASP-26 & $0018-15$ & G0 & 11.3 & 2.76 & 1.02 & 1.32 \\
WASP-28 & $2334-01$ & F9 & 12.0 & 3.41 & 0.91 & 1.12 \\
WASP-29 & $2351-39$ & K4V & 11.3 & 3.92 & 0.24 & 0.79 \\
WASP-30 & $2353-10$ & F8V & 11.9 & 4.16 & 61.0 & 0.89 \\
WASP-31 & $1117-19$ & F7 & 11.7 & 3.41 & 0.47 & 1.56 \\
WASP-32 & $0015+01$ & & 11.3 & 2.72 & 3.60 & 1.18 \\
WASP-34 & $1101-23$ & G5 & 10.4 & 4.32 & 0.59 & 1.22 \\
WASP-35 & & & 11 & 3.2 & & \\
WASP-36 & & & 12 & 1.5 & & \\
WASP-37 & $1447+01$ & G2 & 12.7 & 3.58 & 1.70 & 1.14 \\
WASP-40 & & & 12 & 3 & & \\
WASP-41 & $1242-30$ & G8V & 11.6 & 3.05 & 0.93 & 1.21 \\
\hline & & & & & &
\end{tabular}

$2006 ; 2007)$. The main limitations of the WASP design are the large pixels, often leading to blending, and the "red noise", which usually exceeds photon noise at magnitudes brighter than 12. Red noise is introduced by changes in focus, and by drift of the images over the chips through the night, by colour effects owing to the wide bandpass, and by variations in sky brightness such as through moonlight. For this reason the lightcurves are de-trended prior to the transit search.

\section{Planet discovery}

Follow-up of WASP-South transit candidates involves a major and ongoing collaboration with the Geneva Observatory, whose CORALIE spectrograph on the 1.2-m Euler telescope at La Silla is well matched to the $V=9-13$ brightness of WASP candidates. Euler can also be switched rapidly to a CCD camera, enabling us to obtain photometry of candidates to confirm the reality of transits, and particularly to check for possible blends with nearby stars.

Table 1 lists WASP-South planets as far as WASP-41. WASPs $35,37 \& 40$ were joint discoveries from combined Southern and Northern WASP data, with radial- 


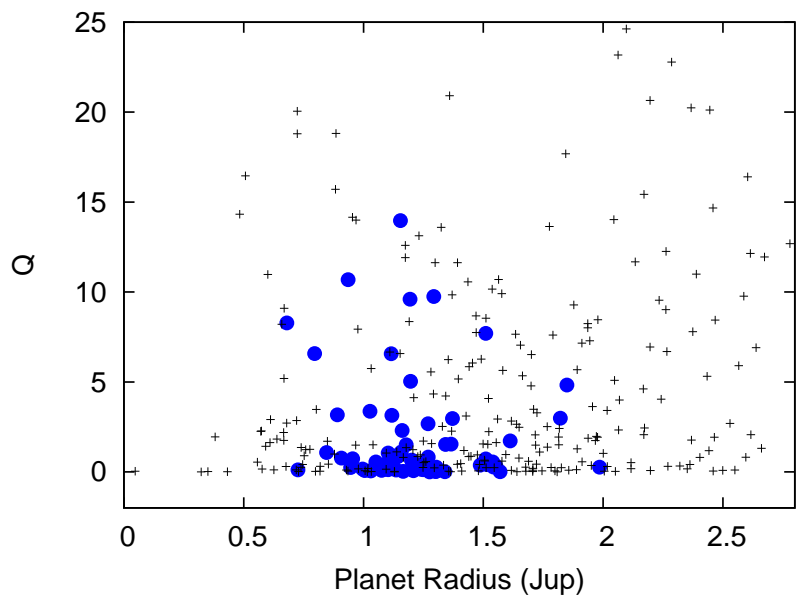

Figure 1: Crosses are the companion radius and $Q$ value for each candidate; circles are the actual planets.

velocity follow-up from OHP and the NOT in addition to CORALIE. Notable finds include the most bloated planet known and the first found in a retrograde orbit, WASP17b (Anderson et al. 2010a); the shortest-period transiting planet, WASP-19b (Hebb et al. 2010); the planet with the strongest known tidal interaction, WASP-18b (Hellier et al. 2009); the highly eccentric 8-day system WASP-8b (Queloz et al. 2010); and the transiting brown-dwarf WASP-30b (Anderson et al. 2010b).

Of the first 485 WASP candidates that were forwarded to Euler, 30 were found to be planets, 54 are still under investigation, and 401 have been eliminated. Thus our overall hit rate is 1 -in-14. The rejected candidates are overwhelmingly astrophysical transit mimics, or blends with stars too close to be resolved in WASP data. Some stars prove to be too hot or fast rotating for CORALIE radial velocities, while for a small number of candidates the transit signal in the WASP data has turned out to be a false alarm.

\section{Candidate selection}

The selection of candidates has evolved over time, and balances hit rate against the desirability of finding unusual planets. For example Fig. 1 shows, for each WASP-South candidate, the planet radius predicted from the WASP data and the Q value, a measure of whether the dip's width and depth is consistent with a planetary transit around a main-sequence star (Collier-Cameron et al. 2007). Both values are only estimates, owing to the low $\mathrm{S} / \mathrm{N}$ and red noise of WASP data.

Candidates with predicted planet radii above $1.6 \mathrm{R}_{\mathrm{Jup}}$ are nearly all transit mimics, as expected, and so have a low hit rate. However, planets such as WASP-17b (Anderson et al. 2010a), with a radius near $2 \mathrm{R}_{\mathrm{Jup}}$, are among our most interesting finds. Candidates with large $\mathrm{Q}$ values are also poor candidates, however a handful do turn out to be planets, for example if the host star is evolved or if the WASP search algorithm has fitted a wrong impact parameter. 


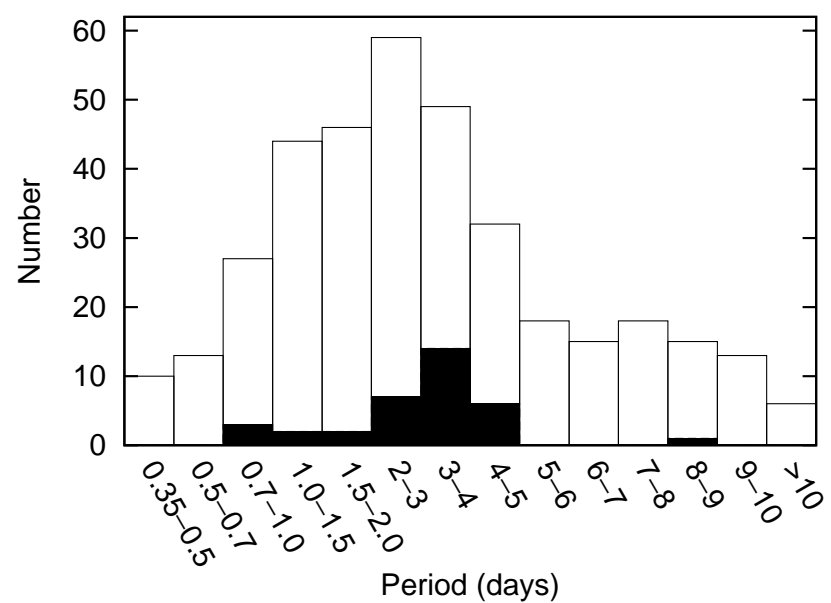

Figure 2: The period distribution of WASP-South candidates compared with that of the resulting planets

Fig. 2 compares the period distribution of the WASP-South candidates with the resulting planets, showing that most of the planets that we find have periods of $2-5$ days. The lower hit rate at periods $0.7-2.0$ days is likely a real phenomenon, since these should be among the easiest planets for WASP to find (and note that HAT has similarly found only one planet below $2 \mathrm{~d}$ ). Above 5 days there are fewer candidates, since the likelihood of covering several transits of any object declines rapidly. The quality of the candidates also declines, since with fewer transits we will be biased towards deeper transits, which have a lower hit rate.

WASP-18b is an example of a planet that is relatively easy for WASP to find: with its short, 0.94-d period, WASP-South covered 16 full or partial transits in its first season, and at $\mathrm{V}=9.3$ the data quality is high (Fig. 3). In contrast, the discovery of the 8.16-d planet WASP-8b was based on only one fairly full and one partial transit (Fig. 4), but, given that it was early days with few WASP-South candidates, that was considered sufficient to justify CORALIE follow-up, even though the period was uncertain and only found from the radial-velocity data.

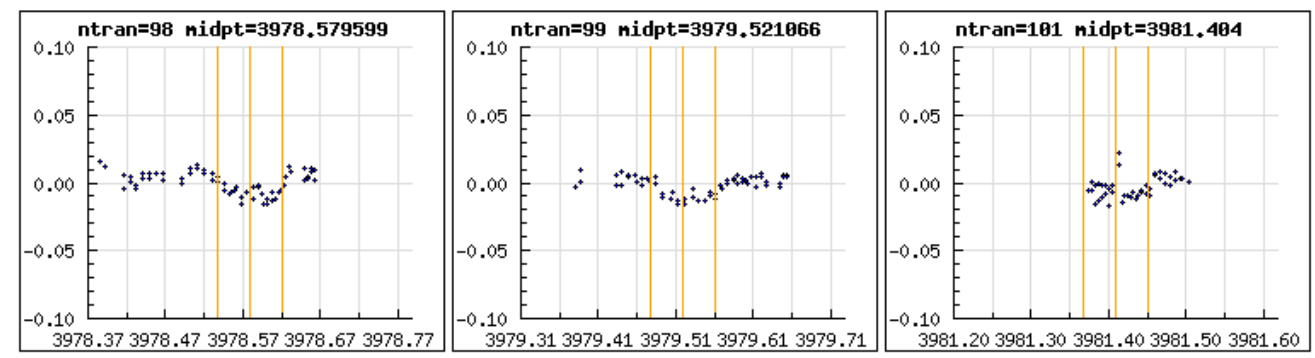

Figure 3: Three of the sixteen discovery transits for WASP-18. 

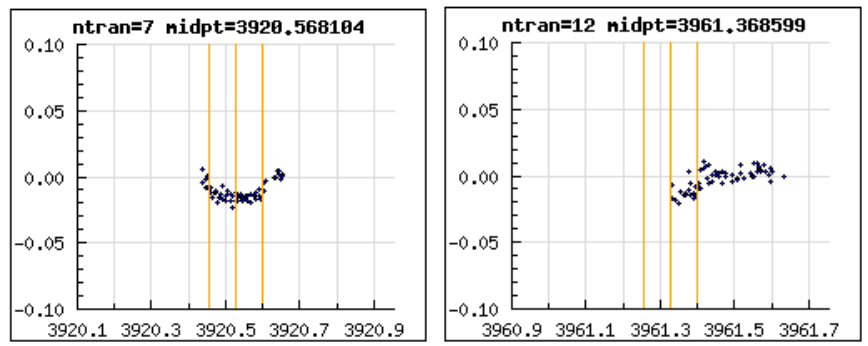

Figure 4: The WASP discovery data for WASP-8.

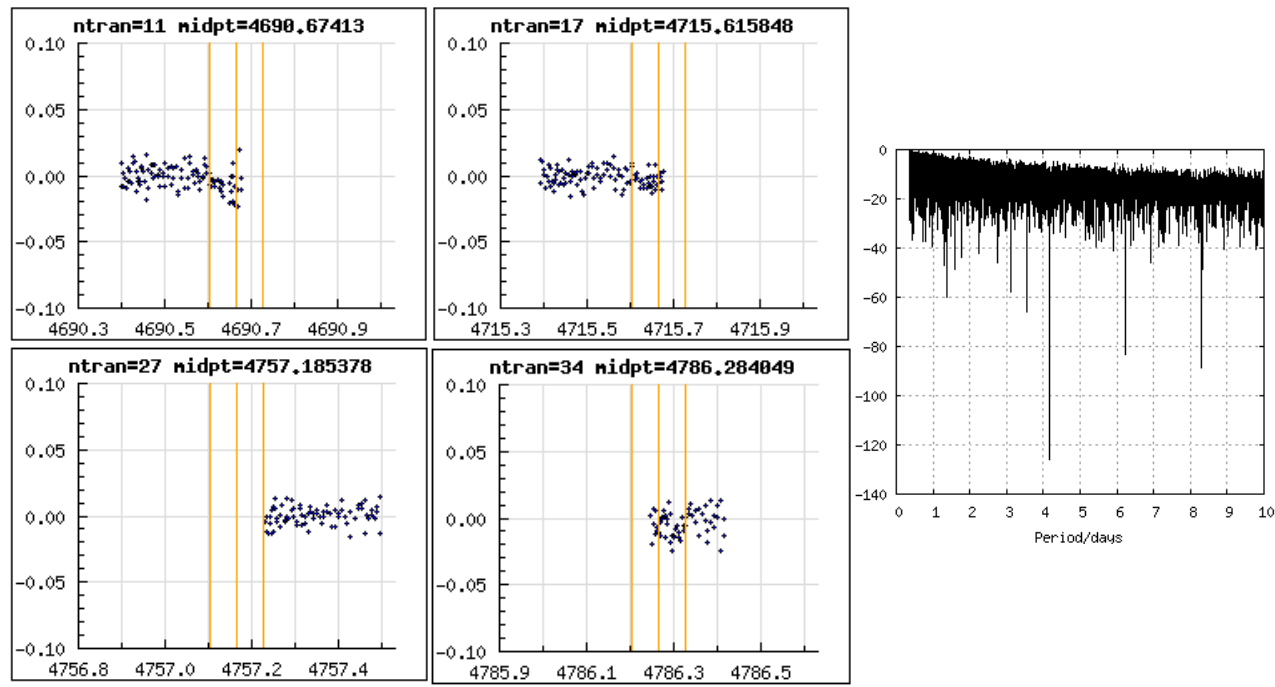

Figure 5: WASP discovery transits and periodogram for WASP-30.

As another example we show in Fig. 5 the discovery data for the brown-dwarf system WASP-30, which has the shallowest transit of the WASP-South discoveries. Both the transit data and the accompanying periodogram are highly marginal, which illustrates the art of selecting candidates from the thousands thrown up by automatic search routines, especially in the presence of red noise which can often mimic transit features.

\section{WASP-South status}

WASP-South discoveries are ongoing, with 180 candidates currently "in play". Clearly it would be possible to markedly improve our hit-rate for finding Hot Jupiters, by using plots such as Figs. 1 \& 2 to select optimum candidates, and by rejecting those with insecure transits or transit periods; however the interest will increasingly be not just in accumulating more $1-M_{\text {Jup }}$ systems in 4-day orbits, but in pushing the envelope 


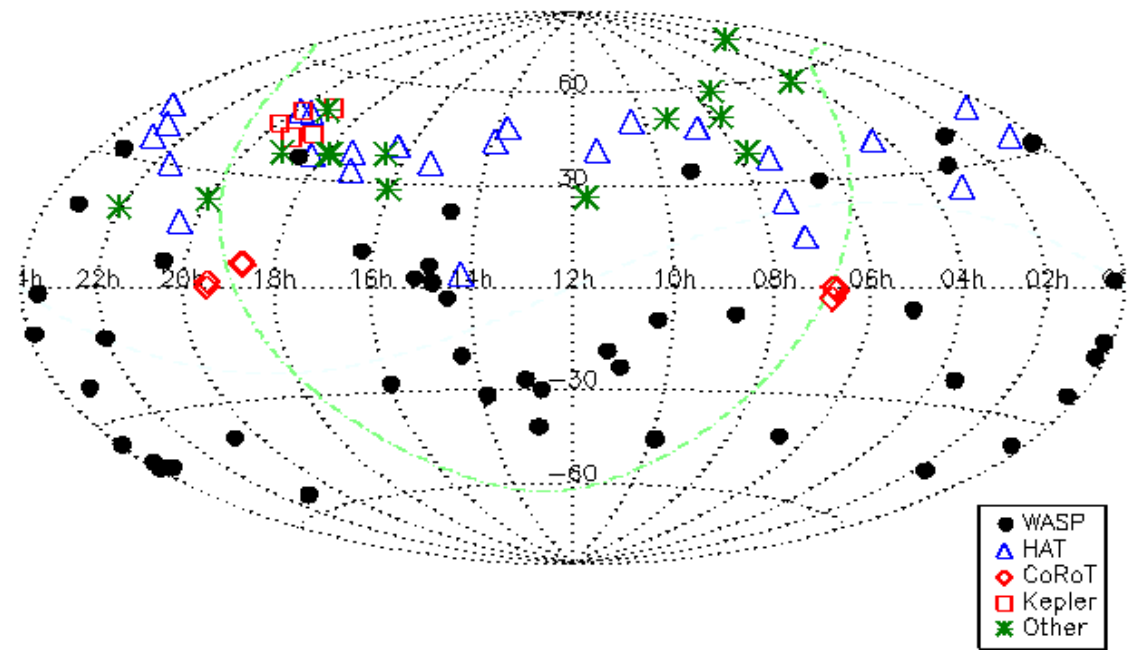

Figure 6: Transiting planets around stars brighter than $V=14$.

of planet parameters, especially by looking for smaller planets that will produce the most marginal transit signals. Although we are running out of virgin sky we expect to continue re-observing the previous regions. Multiple seasons on the same regions of sky will help to overcome red noise, pushing down to shallower transits, while also increasing the likelihood of finding longer-period systems.

The distribution of known transiting exoplanets around bright stars (Fig. 6) shows that the surveys are far from complete: the concentration of known systems near the Kepler field results from the greater concentration of surveys on that region. Extrapolating to the rest of the sky implies that there are several hundred more transiting exoplanets within the discovery capabilities of WASP-like surveys.

Acknowledgements. We thank the South African Astronomical Observatory for hosting WASPSouth and for their ongoing support and assistance.

\section{References}

Anderson, D.R. et al. 2010a, ApJ, 709, 159

Anderson, D.R. et al. 2010b, arXiv:1010.3006

Bakos, G.A. et al. 2004, PASP, 116, 266

Collier Cameron, A. et al. 2006, MNRAS, 373, 799

Collier Cameron, A. et al. 2007, MNRAS, 380, 1230

Hebb, L. et al. 2007, 2010, ApJ, 708, 224

Hellier, C. et al. 2009 Nature, 460, 1098

McCullough P.R. et al. 2006, ApJ, 648, 1228

O'Donovan, F.T. et al. 2006, ApJ, 644, 1237

Pollacco, D.L. et al. 2006, PASP, 118, 1407

Queloz, D. et al. 2010, A\&A, 517, L1

Udalski A. et al. 2002, Acta Astron., 52, 1 